\title{
LA POTENCIACIÓN DEL TURISMO RURAL A TRAVÉS DEL PROGRAMA LEADER: LA MONTAÑA DE ALICANTE
}

\author{
Ana R. Candela Hidalgo \\ $\mathrm{M}^{\mathrm{a}}$ del Mar García Carretero \\ $\mathrm{M}^{\mathrm{a}}$ Paz Such Climent
}

\section{RESUMEN}

El turismo rural se ha planteado como una forma de dinamizar los espacios rurales desfavorecidos, de acuerdo con las premisas del desarrollo local, concebido como un complemento $\mathrm{y}$, en algunos casos, en función de los ámbitos de aplicación, incluso una verdadera alternativa a las rentas agrarias. Estas ideas han venido siendo objeto de atención desde la $\mathrm{CE}$, que ha potenciado el papel del turismo rural a través de diversos programas, entre los que destaca el LEADER. El programa LEADER se convirtió para la Montaña de Alicante en una subvención inesperada y, por tanto, su aplicación ha sufrido las limitaciones que conlleva la falta de experiencia en la gestión y la carencia de una infraestructura básica sin la que se hace difícil sustentar una actividad económica prácticamente inexistente en la zona. El éxito del primer programa LEADER se ha visto condicionado por el restringido período de ejecución, la deficiente difusión de la información, los reducidos porcentajes de subvención y la concentración de las ayudas en las actuaciones realizadas por las entidades públicas.

Palabras clave: turismo rural, LEADER, desarrollo local.

\section{ABSTRACT}

According to the local development premises, rural tourism has been considered as a way for the undeveloped rural spaces improvement. It has been conceived as a complement, and in many cases, in line with the areas where it 
takes place, even as a real alternative to agricultural incomes. These areas have been taking into account by the EC, which has promoted the rural tourism role through several programs. One of the most important is the LEADER. LEADER program was an unexpected subvention for La Montaña of Alicante, and consequently its application has been limited by the inexperience in management and the lack of basic infrastructures. That is why the introduction of a completely new economic activity in the area is very difficult. The succes of the first LEADER program has been conditioned by the short period to carry it into effect, the deficient spread of information, the reduced percentages of the grant and the high concentration of the subventions in the actions which have been carried out by public organisms.

Key words: rural tourism, LEADER, local development

Paralelamente al retorno estacional de ciertos sectores de la población urbana a las zonas rurales con las que mantienen una estrecha vinculación por lazos afectivos, familiares o de procedencia, va tomando cuerpo, con un protagonismo en aumento, la valoración, como alternativa a la oferta turística masificada de sol y playa, de aquellos espacios de alta calidad ambiental, que responde a la mayor apreciación de los valores ecológicos y culturales por un sector creciente de la demanda de origen urbano.

Por otra parte, estas nuevas tendencias han tratado de ser incorporadas con una significación diferente, en función de la época de aplicación y la coyuntura socioeconómica, a las actuaciones que, desde las distintas instancias públicas (comunitarias, nacionales y autonómicas), intentan propiciar un desarrollo equilibrado del territorio que palíe los efectos del modelo de desarrollo tradicional, con especial repercusión en las zonas desfavorecidas de montaña, caracterizadas por el predominio de las actividades primarias y la disminución y el envejecimiento de la población.

\section{El espacio rural en el marco de la Política Agraria Comunitaria. Antece- dentes del Programa LEADER}

En relación al medio rural y, en concreto, a los avatares del sector económico primario, la Unión Europea ha aplicado tradicionalmente en los países miembros, una política de ayuda a la agricultura, basada en la concesión de subvenciones a las producciones, que ha ocasionado con el tiempo serios problemas por la acumulación de excedentes y, por tanto, la consolidación de una agricultura excesivamente protegida y con dificultades para competir en los mercados internacionales. Ante esta situación, la Política Agraria Comunitaria ha sufrido un cambio en sus planteamientos, en un primer momento, en el sentido de disminuir la citada superproducción y favorecer actividades económicas alternativas y, en una segunda fase, 
con la modificación de la PAC, dirigida al abandono del sistema de protección a la agricultura fundamentado en la ayuda a la producción y a la adopción de un sistema de ayudas directo a las rentas.

De acuerdo con esta nueva orientación, la Comunidad ha establecido las bases de su política socioestructural con la que, a la par que reducir los intensos desequilibrios regionales en el seno de los estados miembros, poder amortiguar los efectos negativos de la política de precios en el empobrecimiento de las rentas agrícolas. De hecho, el mantenimiento de un nivel de vida aceptable en las zonas rurales se ha convertido en una de las líneas de actuación de la política comunitaria, en la que también van a incidir objetivos medioambientales.

La Comunidad asume la necesidad de potenciar el desarrollo rural poniendo el acento no tanto en el sostenimiento a toda costa de una agricultura en muchas ocasiones claramente inviable, como en la preservación de las zonas rurales y de su medio ambiente característico. Para ello, resulta imprescindible el establecimiento de unos umbrales mínimos de población que aseguren la continuidad en el paisaje y en el modo de vida tradicional. Si tomamos en consideración la situación socioeconómica de estos espacios, no se puede frenar el despoblamiento ni potenciar el desarrollo rural dependiendo exclusivamente de la agricultura, por lo que la UE insta a la diversificación de la economía de estos espacios. En este sentido, la Comunidad ha previsto la aplicación de distintas medidas e instrumentos financieros destinados a favorecer la introducción de otras actividades económicas, entre las cuales ha destacado el turismo rural como complemento de la actividad agraria tradicional y solución a los problemas de las zonas rurales, con unas expectativas en ocasiones excesivas. Estas reflexiones se ponen de manifiesto en el tratamiento otorgado a las áreas desfavorecidas en el contexto de la evolución de la política estructural comunitaria que pasamos a esbozar.

La UE ha promovido el desarrollo de las áreas rurales y la aparición en las mismas de la actividad turística desde su política agraria y regional a través de la intervención de los fondos estructurales (FEOGA, FEDER y FSE). Por lo que atañe a la política estructural dirigida a favorecer el aumento o, cuanto menos, la conservación de las rentas en el sector agrario, se introducen en la década de los setenta algunas normativas como la Directiva 161/1972 de orientación socioeconómica o profesional que, inspirada en el Plan Mansholt, según el cual el incremento de las rentas rurales pasaba por la reducción del número de activos y el estímulo de actividades económicas no agrarias, contempla la posibilidad de compatibilizar las tareas agrarias con la ocupación turística. También en estas fechas se aprobó la D268/75 de zonas de montaña y áreas desfavorecidas, que establecía un tratamiento especial con posibilidad de recibir indemnizaciones compensatorias, dadas las limitaciones de estos espacios para la agricultura y la importancia de su mantenimiento para salvaguarda de los valores ambientales.

Mayor trascendencia en el marco de la política estructural, contrapunto a la de 
precios y mercados, tendrán, por una parte, la D797/1985 de mejora de la eficacia de las estructuras agrarias que introduce como novedad el concepto de zonas sensibles desde el punto de vista del medio ambiente y, por otra, la puesta en marcha de los Programas Integrados Mediterráneos con ocasión de los Tratados de Adhesión de España y Portugal -D2088/1985- que propugnan un desarrollo rural integrado. Además, la D797/1985 mantiene la indemnización compensatoria en las zonas de agricultura de montaña, a fin de impedir su despoblamiento, y amplía su aplicación a otras áreas para promover prácticas compatibles con la conservación de sus valores paisajísticos y ecológicos, medida con la que por primera vez se introduce la ayuda directa a las rentas en lugar de a la producción. Al mismo tiempo, y de acuerdo con este cambio de orientación de la política estructural, se sigue favoreciendo la diversificación y potenciación de las actividades turísticas como generadoras de empleo y rentas, al incluir ayudas complementarias para zonas de montaña y desfavorecidas. No obstante, se ha de incidir en la escasa repercusión del R.D. 808/1987 (por el que se traspone al derecho español la citada directiva) en la Comunidad Valenciana, puesto que tan sólo se tramitaron cinco expedientes por el concepto de actividades forestales y turístico artesanales para el período 1989-1991, con la concentración de las mismas en el primer año de la serie.

Al objeto de insistir en la reducción de los desequilibrios regionales como condición para el establecimiento del mercado único, se emprende la reforma de los fondos estructurales - Rgto. 2052/88 - , que cobran un mayor protagonismo, a la vez que se suaviza el proteccionismo y se potencia la retirada de cultivos. En esta nueva línea se va a propiciar el desarrollo integral de las regiones más pobres, para las que se articulan los llamados Programas Operativos, con los que se disponen acciones para la protección del medio ambiente y para el desarrollo de infraestructuras rurales, turísticas y forestales con cargo al FEOGA-orientación en las regiones objetivo 1 y $5 b^{1}$. Novedad igualmente es la reserva, a cargo de la Comisión, del $15 \%$ del presupuesto del FEDER para sus iniciativas, una de las cuales es el programa LEADER ${ }^{2}$, del que se espera una incidencia positiva en el estímulo al desarrollo rural y un mayor éxito en la concurrencia de actividades complementarias.

El nuevo rumbo de la política estructural determina también la reforma de la PAC, cuyos objetivos, para la primera mitad de los noventa, son lograr la presencia de un número suficiente de agricultores en las zonas rurales, como garantía de preservación del medio ambiente, y el fomento de actividades económicas fuera del sector agrario como base para el desarrollo del medio rural. Se impulsa por

1 El objetivo 1 afecta a aquellas zonas que presentan un PIB per cápita inferior al $75 \%$ de la media comunitaria, mientras que el objetivo $5 \mathrm{~b}$ va dirigido al desarrollo de las zonas rurales.

2 Relaciones entre actividades de desarrollo de economía rural. 
tanto, ante la imposibilidad de modernizar la agricultura en algunas zonas, el cese de la producción agraria y, con la finalidad de proteger el medio ambiente, el compromiso de los agricultores de destinar sus tierras a repoblación forestal o creación de reservas ecológicas.

Se comprende así, de acuerdo con Eladio Arnalte, la distinción de dos tipos de espacios rurales, las áreas que se mantienen en explotación y aquellas otras que se destinan a espacios de ocio de la población urbana. Estos últimos, en su casi totalidad, se reparten en los países que conforman la periferia europea y son bañados por el mar Mediterráneo — excepto Irlanda-, por lo que cuentan con favorables condiciones climáticas para la práctica de actividades recreativas.

\section{El turismo rural en la Comunidad Valenciana}

Las primeras actuaciones dirigidas al fomento del turismo rural en España se asocian al programa de Casas de Labranza, patrocinado por el Servicio de Extensión Agraria y la Subsecretaría de Turismo que, iniciado en 1967, mantuvo una vigencia cercana a dos décadas. Otras iniciativas de carácter más puntual, en las que ya se contempla el papel del turismo rural en el desarrollo local con una visión integrada, se llevan a cabo durante la primera mitad de los años ochenta en las comunidades de Aragón, Extremadura, Asturias y Valencia.

Con carácter general se promulga una normativa específica para las áreas de montaña, la Ley 25/1982, que preveía dentro del régimen de ayudas una línea de crédito para la financiación de actividades complementarias artesanales y turísticas y contemplaba la elaboración de Programas de Ordenación y Promoción de dichas zonas, a cargo de la Conselleria de Agricultura en el caso de la Comunidad Valenciana. Este organismo, al igual que sus homólogos en otras comunidades autónomas, fue el encargado de delimitar las posibles zonas de montaña. La establecida en la provincia de Alicante afectó, en un principio, a cuarenta y cuatro municipios pertenecientes a diferentes comarcas, con un mayor volumen de población y porcentaje de SAU en relación a las restantes zonas de montaña de Castellón y Valencia. Posteriormente, se han englobado nuevos municipios dentro de las zonas de montaña de forma que ascienden a un total de ciento cincuenta y ocho en el conjunto del territorio valenciano y a cuarenta y seis municipios en Alicante ${ }^{3}$. En el correspondiente PROPROM se consideraba el turismo entre las actividades a promover. Sin embargo, estos programas no llegaron a ser ejecutados, entre otras razones, por la falta de coordinación entre las distintas administraciones sectoriales involucradas y las dificultades de financiación. No obstante, y con motivo de la puesta en marcha de la iniciativa comunitaria del programa LEADER, con una

3 Está pendiente de aprobación la integración en zonas desfavorecidas de montaña de catorce nuevos municipios colindantes a los actualmente declarados. 
dotación de 400 millones de ecus, fue la propia Conselleria de Agricultura la que aprovechó las líneas directrices de actuación apuntadas en los citados PROPROM para presentar su solicitud, de manera que fueron aprobados por la UE tres proyectos dentro del Programa LEADER con una duración prevista de 4 años (19901993): Els Ports, Alto Mijares-Alto Palancia en Castellón y La Montaña en Alicante.

Antes de pasar al análisis más detallado del programa LEADER en La Montaña de Alicante, repasamos brevemente la política turística diseñada por la Conselleria de Industria, Comercio y Turismo en relación con el turismo de interior a partir de 1990. Cabe citar, asimismo, que ya se habían puesto en marcha algunas experiencias de agroturismo amparadas por el Instituto de Cooperativismo Agrario en Valencia y Castellón, al objeto de favorecer esa diversificación de las actividades económicas en la lógica de los cambios operados en la PAC. El Instituto Turístico Valenciano (organismo dependiente de la Conselleria de Industria, Comercio y Turismo) definió, en el Libro Blanco del Turismo de la Comunidad Valenciana, el Plan Estratégico ETVA 2001, en el que se incluye un Plan General de Productos Turísticos, más tarde denominado Plan de Diversificación de la Oferta Turística. En este último se configura un Programa de Turismo Interior, cuyos objetivos son la captación de nuevos mercados y la desviación de los flujos e inversiones turísticas a zonas no litorales a fin de dotarlas de una oferta hasta ese momento inexistente. Al mismo tiempo, el ITVA — dentro de sus programas de apoyo al sector turístico, en concreto en el Programa de Apoyo a las Inversiones- concede ayudas a la creación de nuevos establecimientos turísticos para promocionar la oferta en zonas de interior.

Por lo que atañe al turismo interior, aunque ya existe alguna actuación basada en la promoción de iniciativas municipales durante 1990 y 1991, el programa como tal aparece en 1992, pero dirigido a la creación de una red de establecimientos de titularidad pública y gestión privada. Las dificultades suscitadas por la escasa capacidad técnica y financiera de los ayuntamientos limitaron el éxito de aquellas primeras tentativas. Desde entonces, la intervención directa del ITVA se ha centrado en tres líneas principales: creación y modernización de alojamientos turísticos, creación y modernización de oferta complementaria, y protección y conservación del medio ambiente y el patrimonio inmobiliario de valor cultural, aunque ha dominado el carácter disperso y puntual de las intervenciones. A este respecto, sólo es reseñable la concentración de actuaciones, que dan lugar a una oferta más completa, en las comarcas castellonenses de Els Ports-Alt Maestrat y Alto Palancia, como se desprende del análisis de las memorias anuales de la citada Conselleria. De una cantidad próxima a los 1.826 millones de pesetas invertidos en la Comunidad Valenciana, correspondieron a la provincia de Alicante más de 348 millones, destinados a la creación de dos hoteles en Biar y Gaianes, un camping en la Vall d'Alcalà y otras intervenciones de menor relevancia en Elx y Callosa d'En 
Sarrià. No obstante, para el trienio 1994-96, está presupuestada una cantidad superior a los 500 millones de pesetas.

\section{La aplicación del Programa LEADER ${ }^{4}$ en la provincia de Alicante}

Con un total de cincuenta y dos programas aprobados en el conjunto nacional y una dotación comunitaria de 120 millones de ecus (equivalentes en el momento de la aprobación a 15.600 millones de pesetas), España se constituye en el país más favorecido por esta iniciativa. La partida asignada a la Comunidad Valenciana asciende a 1.131 millones, de cuya distribución resulta particularmente beneficiada La Montaña de Alicante, con 465 millones $^{5}$ (y una inversión global estimada en 1.557,5 millones), el segundo leader en volumen de subvención a nivel nacional, sólo superado por el de Urbión en la Comunidad de Castilla y León.

Dentro de estos programas, el turismo rural es la línea que más recursos monetarios ha acaparado, con un $51,7 \%$ del presupuesto global, siguiendo una tendencia que se aprecia, asimismo, en el resto de grupos europeos, fundamentalmente en los situados en el espacio mediterráneo. Este ámbito concentra la mayor parte de las regiones definidas por la Comunidad como objetivo 1 y $5 b^{6}$, zonas a las que va dirigido el LEADER. Es, pues, la iniciativa comunitaria desde la que se han destinado las mayores inversiones al turismo rural, con subvenciones que oscilan entre el $35 \%$ en las áreas incluidas en regiones 5 b y el $50 \%$ en las de objetivo 1.

La Comisión Europea concibe el programa LEADER como una iniciativa demostrativa de desarrollo rural que pretende aportar soluciones innovadoras, desde el equilibrio y diversificación de sus actividades económicas y el mantenimiento del tejido social, que puedan servir de modelo para otras zonas rurales, a través de la potenciación de los recursos propios y la participación directa de los agentes locales. Se entiende así, de acuerdo con la reciente concepción del desarrollo local, que la viabilidad de estas zonas radica en su desarrollo endógeno. En este contexto, se enmarca la creación de una red de grupos de acción local encargados de la gestión y puesta en funcionamiento del programa, en los que se dé cabida a agentes económicos y sociales tanto públicos como privados.

El texto comunitario especifica que las medidas subvencionables, dentro de las estrategias que presenten cada uno de los grupos, son las orientadas al desarrollo

4 (doc. SEC(90) 1602 final). CE C73/ 14, D.O.C.E. de 19 de marzo de 1991.

5 La cuantía del total de subvención Leader se establece en función de las variaciones de cotización de la peseta con repecto al ECU, lo que justifica las diferencias observadas en las cantidades recogidas en el texto.

6 Estas regiones comprendían el 55\% del territorio de la Comunidad en el momento de la asunción de la iniciativa LEADER por la Comisión de las Comunidades Europeas. Se exige en estas zonas una población comprendida entre los 5.000 y los 100.000 habitantes para la aplicación de este PIC. 
rural propiamente dicho, las relativas a la creación, equipamiento y gestión de los grupos y, por último, al establecimiento de una red supranacional de grupos de acción local. No cabe duda que el contenido de los planes propuestos se ha centrado en el primero de los epígrafes mencionados: apoyo técnico al desarrollo rural, formación profesional y ayudas al empleo, turismo rural, respaldo a las pequeñas empresas, a la artesanía y a los servicios locales y valorización y comercialización in situ de la producción agraria, entre otros.

\subsection{La Montaña de Alicante}

Antes de acometer una breve descripción de la zona, se hace necesario incidir en la acentuada dualidad demográfica y territorial que existe entre el interior provincial y el litoral fuertemente urbanizado, que concentra la mayor parte de la población y las principales actividades económicas.

Un carácter supracomarcal y una cierta homogeneidad física y humana reflejada en un modelo peculiar de ocupación del espacio, definen el ámbito geográfico delimitado como zona de montaña, que engloba cuarenta y cuatro municipios y abarca una superficie de $1.043 \mathrm{Km}^{2}$ (el $18 \%$ de la superficie provincial), en la que residen 35.641 habitantes (aproximadamente el 2,75\% del total provincial), dando lugar a unas bajas densidades de población (ver fig. 1).

El relieve, constituido por estructuras y litofacies asociadas al Prebético alicantino, se dispone en una alternancia de sierras de altitud superior a los $1.000 \mathrm{~m}$. y valles constreñidos por ellas, de dirección SW-NE, que configuran un espacio fragmentado y contrastado, con pendientes muy acusadas (entre el 20 y $50 \%$ ).

Las principales vías de penetración, la carretera N-340 Alicante-Alcoy-Valencia y la comarcal 3318 Benidorm-Oliva, transversales ambas al relieve, determinan los principales ejes de crecimiento económico y de dinámica poblacional. Así, la primera de ellas, desde Alcoy, focaliza y difunde la actividad industrial en el sector occidental, sobre todo en los municipios de Cocentaina y Muro. La segunda, cuyo núcleo más destacado, Callosa d'En Sarrià7, basa su actual desarrollo económico en una agricultura altamente competitiva, ha canalizado tradicionalmente la irradiación del turismo de Benidorm a los pueblos vecinos de segunda línea como Polop y La Nucía, al tiempo que ha facilitado el acceso a los municipios del valle del Guadalest, convertidos en un complemento de la oferta turística de Benidorm. A éstas se añaden las posibilidades que ofrece la apertura de un enlace de Alicante con Alcoy a través de la Hoya de Castalla que evita el Puerto de la Carrasqueta.

Al margen de estos sectores, el resto de la zona de montaña, cuyo acceso se realiza a través de corredores paralelos a las principales sierras, presenta mayores

7 Polop y Callosa d'En Sarrià, calificadas como zona de montaña tras la aprobación del primer LEADER, fueron excluidas del mismo; aunque se han incorporado en la propuesta presentada al LEADER II. 


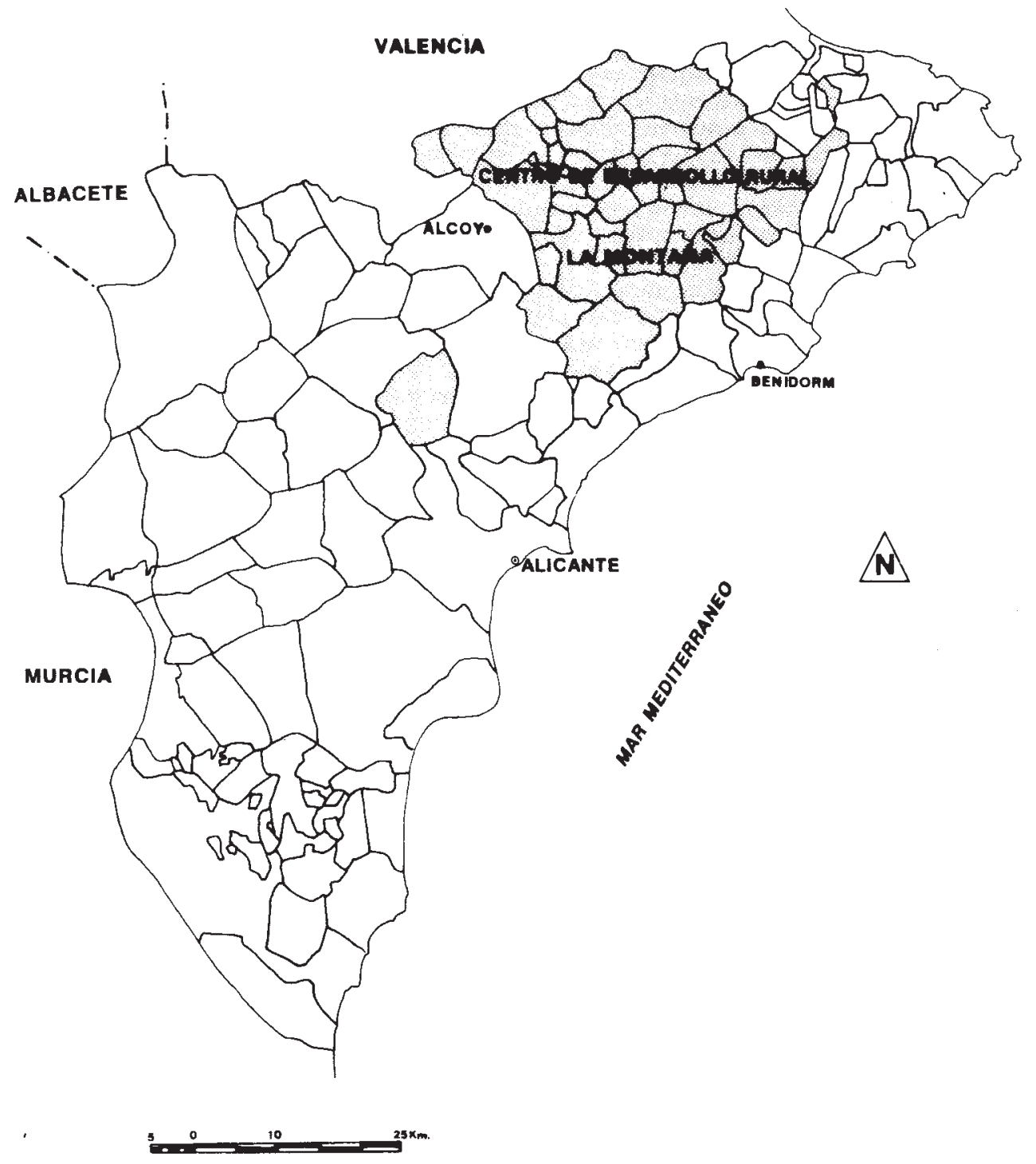

Figura 1. Localización geográfica del área de estudio.

dificultades de comunicación, de las que deriva su relativo aislamiento, teniendo en cuenta su proximidad a la costa que, de alguna manera, matiza su definición como espacio de interior. Es en esta última donde se concentran los mayores porcentajes de población activa agrícola, superiores al $60 \%$, aunque ligada a una agricultura de secano escasamente tecnificada, con una inadecuada estructura de las explotaciones y una elevada edad media de los titulares de las mismas que 
condicionan la falta de competitividad y la inviabilidad económica de esta agricultura. No extraña, pues, que sea este área la que experimenta una regresión demográfica más acusada, producto de la continuada corriente emigratoria dirigida primordialmente hacia las zonas turísticas del litoral y los centros industriales del interior, que ha repercutido en el deterioro del patrimonio inmobiliario, cultural y paisajístico.

No obstante, la Montaña de Alicante conserva todavía mayores efectivos demográficos que el resto de las zonas de montaña de la Comunidad Valenciana, en parte por las posibilidades de desplazamiento hacia los centros de servicios y mercados laborales próximos, manteniendo la residencia en sus núcleos de origen. La crisis de la actividad económica tradicional ha hecho necesaria la búsqueda de alternativas de desarrollo local que proporcionen un complemento a los ingresos de la población, entre las que destaca el aprovechamiento de los potenciales recursos turísticos de este espacio; actuaciones que se inscriben en el marco conceptual esbozado en líneas anteriores. Se trataría, por tanto, de poner en valor, además de la belleza propia del paisaje montañoso y las posibilidades de ocio de una orografía accidentada, la riqueza de su patrimonio histórico-artístico y cultural (arte rupestre y yacimientos arqueológicos, fortificaciones árabes, despoblados moriscos y los pozos de nieve característicos de la economía de esta región en épocas pasadas). Tal variedad de recursos puede contribuir a consolidar, por una parte, el turismo de retorno y el de estancias cortas aprovechando la proximidad de los núcleos del litoral, a la vez que potenciar la captación de otros segmentos de la demanda atraídos por el contacto con la naturaleza, bien bajo la fórmula de turismo rural o de turismo de aventura relacionado con prácticas deportivas. Es precisamente a la consecución de estas metas a las que se ha enfocado la aplicación del programa LEADER en La Montaña de Alicante.

\subsection{Análisis y valoración del programa LEADER de La Montaña de Alicante}

En lo que atañe a la aplicación de las subvenciones, el Programa LEADER contempla únicamente su concesión bajo la forma de subvención de capital. Destaca singularmente, entre las medidas consideradas, el turismo rural, aunque la artesanía, los servicios locales o la comercialización de la producción agraria no dejan de tenerse en cuenta. Los porcentajes estipulados para las medidas mencionadas, que pueden alcanzar, en función de las líneas, un 40 ó 50\% de subvención en cada proyecto en las regiones objetivo 1, en el caso de la Montaña alicantina no se cumplen más que cuando las actuaciones son emprendidas por entidades públicas o asociaciones sin ánimo de lucro.

Los umbrales máximos de ayuda leader que puede recibir un proyecto se establecen en 20 millones de pesetas, excepto cuando afectan a la rehabilitación 
del patrimonio histórico-artístico y a la recuperación de parajes naturales ${ }^{8}$, y de 50 millones si a la cantidad facilitada por el programa europeo se añaden las proporcionadas por las diferentes administraciones de los Estados miembros ${ }^{9}$, ya que este programa permite la compatibilización de sus subvenciones con las de otros organismos estatales y autonómicos, en un intento de favorecer la colaboración entre distintas instancias y potenciar la sinergia que de este hecho se puede derivar. A este respecto, algunos beneficiarios, asesorados por el CEDER, han tratado de completar las ayudas mediante la inclusión de sus proyectos en líneas paralelas y complementarias acometidas por organismos como el ITVA (Institut Turistic Valencià) o el IMPIVA (Instituto para la Pequeña y Mediana Empresa Valenciana) ${ }^{10}$, que en todo caso no han rebasado el $10 \%$ de la inversión ${ }^{11}$; puesto que, en lo concerniente al leader de La Montaña, los porcentajes de subvención raramente han superado el $30 \%$ en proyectos presentados por particulares, a todas luces insuficientes a la hora de promover la creación de empresas en un ámbito rural tan reacio a cambios bruscos y a emprender actividades económicas con un alto componente de riesgo, máxime si se añade la intrínseca falta de capitales. Hecho que puede justificar el predominio de las actuaciones destinadas a la ampliación o mejora de negocios ya existentes, que han aprovechado la coyuntura ofrecida por el LEADER para llevar a la práctica, en condiciones más ventajosas, proyectos previstos con anterioridad. No es este el caso de los ayuntamientos que han percibido hasta un $50 \%$ del presupuesto de inversión.

Los beneficiarios, además, están obligados a comunicar la aceptación de la concesión y adjuntar la documentación sobre el presupuesto de inversiones, la finalidad del mismo y los plazos de ejecución, así como la justificación del gasto (mediante verificación del proyecto o memoria valorada en los activos fijos y facturas en el caso de bienes inmuebles), una vez que la inversión haya sido finalmente realizada y se solicite el pago de la subvención.

Como en el resto de los proyectos LEADER en España, el de La Montaña comenzó su andadura tras la firma de un convenio entre el Centro de Desarrollo Rural creado al efecto y el IRYDA, organismo encargado de coordinar y supervisar la ejecución del programa en el ámbito estatal. Este convenio, suscrito en abril de 1992, sentó las bases para su aplicación, ajustadas a lo que el PIC europeo había establecido al respecto. Destaca el compromiso del IRYDA de anticipar como máximo el $40 \%$ del presupuesto global a la firma del convenio, previa presenta-

8 En este caso, los límites de ayuda alcanzan los 25 millones de pesetas.

9 Convenio suscrito entre el IRYDA y el CEDER de La Montaña.

10 Las ayudas paralelas solicitadas se han acogido al programa de apoyo a la inversión «modernización/creación de establecimientos turísticos» del ITVA que, a su vez, ha desviado algunos proyectos a líneas similares acometidas por el IMPIVA.

11 Únicamente en 1993 se aceptaron algunas de las solicitudes tramitadas desde el CEDER, práctica que, sin embargo, no ha tenido continuación en 1994. 
ción de los avales correspondientes ${ }^{12}$, y de satisfacer la cantidad restante hasta completar un $80 \%$ tras la justificación del pago a los beneficiarios finales de, al menos, un $50 \%$ de las subvenciones concedidas. Sin olvidar, asimismo, la obligación del CEDER de enviar memorias anuales sobre sus actividades.

Si bien en un primer momento la Conselleria de Agricultura intervino activamente en la génesis del leader La Montaña, su presencia fue diluyéndose a medida que tomaba cuerpo la asunción de responsabilidades por los centros de gestión local. El CEDER de La Montaña se convirtió, por tanto, en el organismo, integrado por administraciones públicas de carácter local, encargado de gestionar la subvención europea y dar a conocer las posibilidades de la aplicación de la iniciativa comunitaria a los municipios integrados en la misma, mediante la difusión de las normas del programa tanto a los ayuntamientos como a los particulares. El núcleo original del CEDER estaba constituido por cinco municipios, Cocentaina, L'Alqueria d'Asnar, La Torre de les Maçanes, Planes y L'Orxa, a los que posteriormente se unirían Fageca y Alcosser de Planes. La sede se ubicó en Cocentaina, el municipio que presenta los mayores niveles de población, desarrollo económico e infraestructural y, por tanto, en principio, con la capacidad suficiente para acoger un centro de estas características. Sin embargo, se ha comprobado que la escasez de recursos materiales y humanos es una de las principales dificultades surgidas a la hora de afrontar la gestión de una subvención de tal magnitud, planteándose, de este modo, contradicciones entre el protagonismo otorgado por el LEADER a la participación de los agentes locales y la capacidad resolutiva de los mismos, a la que se añade la escasa presencia de una trama previa de asociaciones de desarrollo local que hubiera podido actuar de soporte básico aportando su experiencia en este campo. Por este motivo, quizás hubiera sido necesaria la intervención de un organismo provincial o autónomico en la gestión (opción adoptada en otras áreas), al menos, en las primeras fases. A estas dificultades se suma el breve período previsto para la ejecución del programa al concebirse como experiencia piloto.

Aunque inicialmente se consideró como plazo límite para la concesión de ayudas el 31 de diciembre de 1993, el IRYDA aprobó su ampliación hasta el 30 de abril de 1994, prorrogándolo de nuevo a septiembre del mismo año a fin de incluir aquellos proyectos que se mantenían en reserva, en caso de producirse renuncias o existir fondos sobrantes, y fijando la fecha límite de ejecución de todos los proyectos presentados en abril de $1995^{13}$.

12 En este contexto, se ha de situar el apoyo de la Diputación Provincial que ha contribuido a los gastos de funcionamiento del CEDER y ha facilitado el soporte financiero para la obtención de los avales.

13 Está prevista la revisión y actualización de los datos manejados en el presente artículo, puesto que los mismos van referidos a fechas anteriores a la finalización de la aplicación del programa y al cierre del ejercicio económico correspondiente. 
Grafico 1. Relación de proyectos subvencionados y denegaciones/renuncias por municipio.

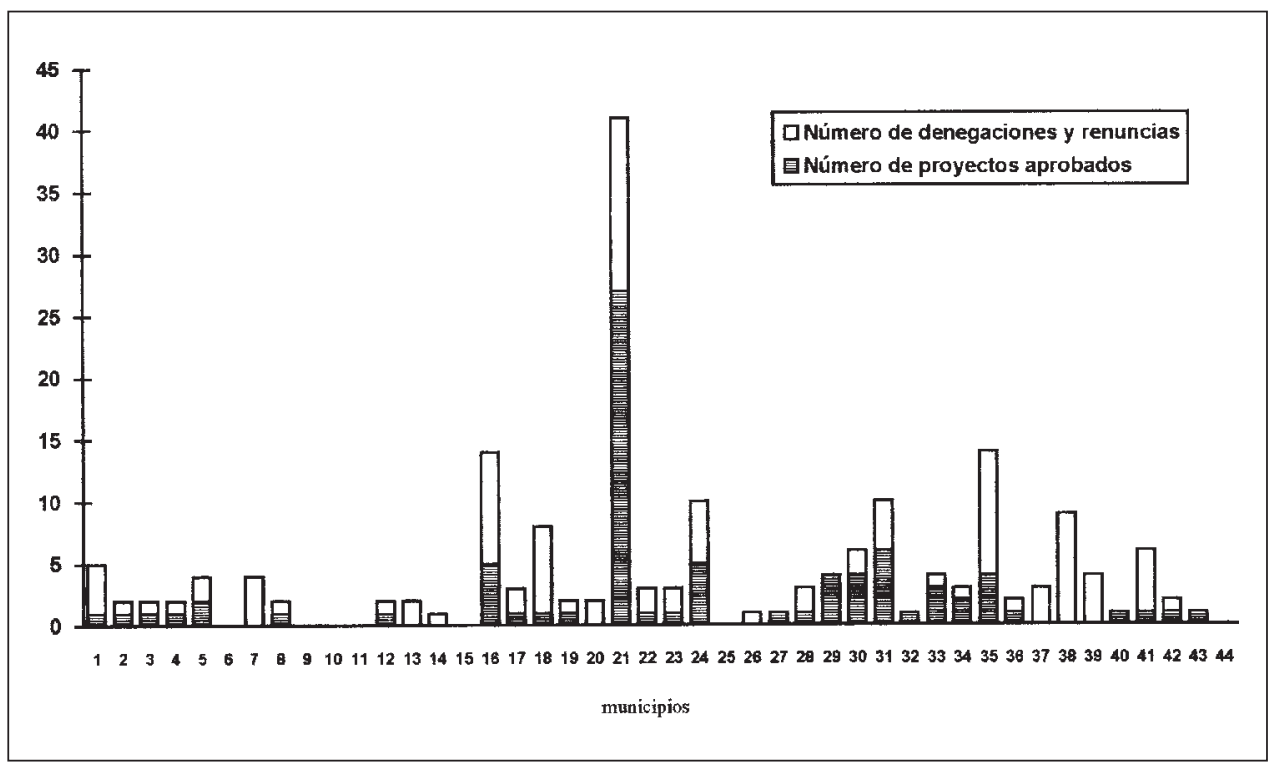

Fuente: Centro de Desarrollo Rural La Montaña. Datos a 30 -IX-1994. Elaboración propia.

En total, fueron presentadas cerca de 200 solicitudes, de las cuales se aprobaron 83, entre las que se incluyen algunas de las consideradas inicialmente en reserva, a tenor del número de renuncias producidas. De los 44 municipios afectados, quince no registran ninguna actuación, a pesar de que ocho de ellos habían presentado solicitudes (ver gráf. 1). Ante resultados de experiencias similares, los gestores del CEDER esperaban conseguir un porcentaje superior al $70 \%$ de proyectos totalmente ejecutados.

El mayor número de solicitudes presentadas correspondió a la medida 3 (turismo rural), medida 4 (PYMES, artesanía y servicios) y medida 5 (valorización y comercialización de productos agrarios); predominando claramente en el cómputo final de proyectos aprobados los orientados al turismo rural, que acumularon el $87,68 \%$ de la subvención comunitaria, dato que contrasta fuertemente con los valores alcanzados en las otras dos medidas mencionadas, 3,31\% y 1,27\% respectivamente (ver gráf. 2).

Según el programa previsto originalmente, tras las primeras estimaciones, al turismo rural le correspondía el 75\% de los 474,6 millones de pesetas de ayuda comunitaria, mientras que la inversión privada en este apartado se situaba en un $40 \%$. Estos valores se incrementarían una vez efectuada la definitiva selección de los proyectos, hasta el $87,68 \%$, ya mencionado, en el primer caso y el $67,4 \%$ en el segundo. El aumento del gasto privado se relaciona con la disminución experimentada, tanto en los porcentajes de subvención comunitaria estipulados que se redu- 


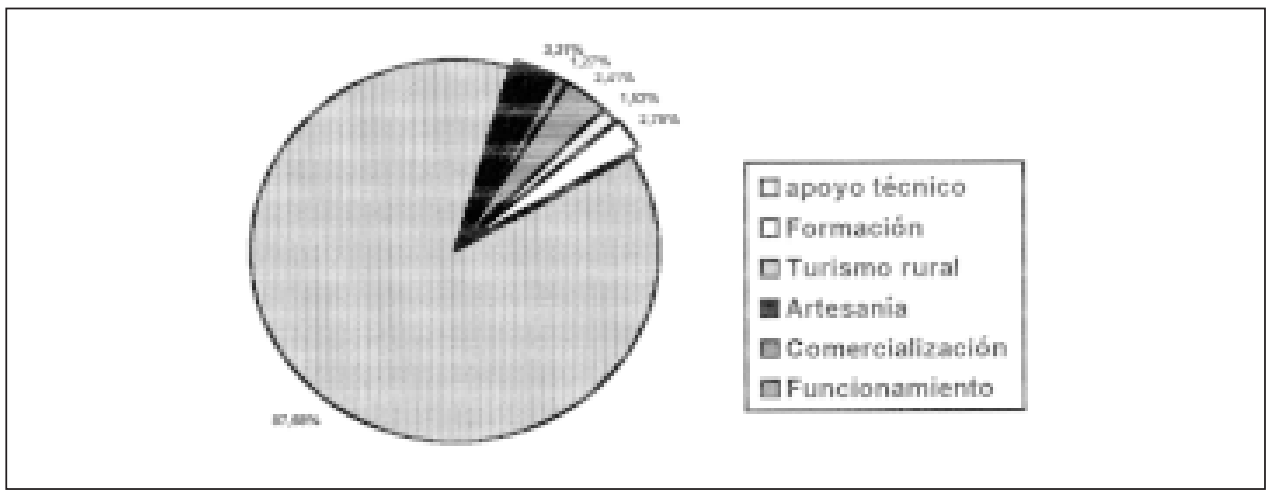

Fuente: CEDER. Datos a 30-IX-1994. Elaboración propia.

cen de los compromisos iniciales del 40\%, a unos porcentajes finales que oscilan entre el $10 \%$ y $25 \%$, como en el gasto previsto a cargo de la administración autonómica.

Dentro del capítulo de turismo rural, para el que está previsto que genere una inversión de 1.328,6 millones de pesetas, de los que 416 millones corresponden a la subvención leader, las actuaciones comprendidas han abarcado desde la promoción turística, oferta complementaria, alojamiento y restauración, hasta la rehabilitación del patrimonio histórico-artístico y la recuperación y acondicionamiento de parajes naturales. Los criterios de valoración adoptados por el CEDER han primado la creación de una oferta de alojamiento, prácticamente inexistente en la zona, así como los servicios de restauración que han supuesto en el primer caso el $30,19 \%$ de las actuaciones y el 28,7 \% en el segundo. En este sentido, la oferta de alojamiento ha acaparado 488 millones, es decir, más de la tercera parte de la inversión en turismo rural y, asimismo, el 33,3\% de la subvención leader. En esta línea, han sido las pensiones y los albergues las modalidades más potenciadas frente a la rehabilitación de viviendas y zonas de acampada. Es interesante reseñar al respecto que solamente se ha acometido la rehabilitación de dos viviendas en el núcleo de población de Fageca, y con carácter experimental, desdeñándose además la adecuación para este fin de otro tipo de alojamientos rurales (acampada en finca particular, masías o vivienda compartida con los propietarios), a diferencia de otras comunidades autónomas donde estas opciones sí adquieren relevancia. Por otra parte, la restauración, con una inversión superior a los 358 millones de pesetas (27,01\% de la inversión en turismo rural) consigue el $18 \%$ de los fondos leader. Ambas líneas de actuación han sido realizadas tanto por particulares como por asociaciones sin ánimo de lucro y ayuntamientos. Estos últimos mantienen un protagonismo exclusivo en lo que se refiere al acondicionamiento y conservación de parajes naturales y patrimonio histórico-artístico. Por el contrario, apenas se 
han efectuado otras intervenciones de gran interés para la puesta en valor del potencial turístico de estas zonas, como las encaminadas a la promoción o a la dotación de oferta complementaria y definición de productos turísticos específicos.

Tabla 1

DISTRIBUCIÓN DE LA INVERSIÓN Y DE LA SUBVENCIÓN LEADER EN TURISMO RURAL (Datos porcentuales)

\begin{tabular}{|lrcccc|}
\hline PROYECTOS & INVERSIÓN & $\begin{array}{c}\text { PROYECTOS } \\
\text { SUBVENCIONADOS }\end{array}$ & $\begin{array}{c}\text { SUBVENCIÓN } \\
\text { LEADER }\end{array}$ & $\begin{array}{c}\text { SUBVENCIÓN } \\
\text { PARTICULARES* }\end{array}$ & $\begin{array}{c}\text { SUBVENCIÓN } \\
\text { AYTO./ASOC.* }\end{array}$ \\
\hline RESTAURACIÓN & 27,01 & 28,57 & 18,02 & $10-22$ & $33,8-40$ \\
PENSIÓN-HOSTAL & 18,40 & 12,7 & 13,39 & $17,6-28,7$ & 50 \\
ALBERGUES & 15,09 & 11,11 & 16,56 & 20 & $32-50$ \\
REHAB. VIVIENDAS & 2,33 & 3,17 & 2,53 & 24,90 & 40 \\
ZONAS ACAMPADA & 0,90 & 3,17 & 1,15 & & 40 \\
REHAB. PATRIMONIO & 12,23 & 6,35 & 15,38 & & $18,84-50$ \\
PARAJES NATURALES & 10,86 & 11,11 & 16,76 & & $40-50$ \\
MUSEOS & 6,34 & 6,35 & 7,22 & & $22,5-40$ \\
CULTURA Y OCIO & 4,52 & 3,17 & 5,53 & & $30-40$ \\
PROMOCIÓN & 1,75 & 11,11 & 2,55 & & $20-60$ \\
SEÑALIZACIÓN & 0,57 & 3,17 & 0,91 & & 50 \\
\hline
\end{tabular}

* Intervalos de subvención. Fuente: CEDER. Datos a 30-IX-1994. Elaboración propia.

Se ha de destacar, en relación a estas matizaciones, las considerables diferencias en los porcentajes de subvención concedidos a ayuntamientos y asociaciones frente a los otorgados a la empresa privada que, en algunos casos, han tratado de paliar las desfavorables condiciones de partida y la falta de iniciativa en ciertos municipios $^{14}$, generalmente de reducido presupuesto (ver tabla 1). Este hecho también se refleja en el cuadro anexo en el que se pone de manifiesto la elevada cuantía de las inversiones y subvenciones que corresponden a las corporaciones locales en las distintas líneas de turismo rural (ver tabla 2). En este sentido, los ayuntamientos, que han ejecutado más de un tercio de los proyectos, han concentrado casi el $40 \%$ de la inversión en turismo rural, a la que ha correspondido un $53,47 \%$ de la subvención leader, porcentajes que se acentúan si a éstos se añaden las iniciativas presentadas por organizaciones sin ánimo de lucro.

14 En entidades de población como Vall d'Alcalà, Millena, Tollos, Alfafara y Benimassot en las que la falta de accesibilidad y el escaso dinamismo económico determinan la inexistencia de oferta, las subvenciones han alcanzado, generalmente, los máximos límites de ayuda permitidos. 


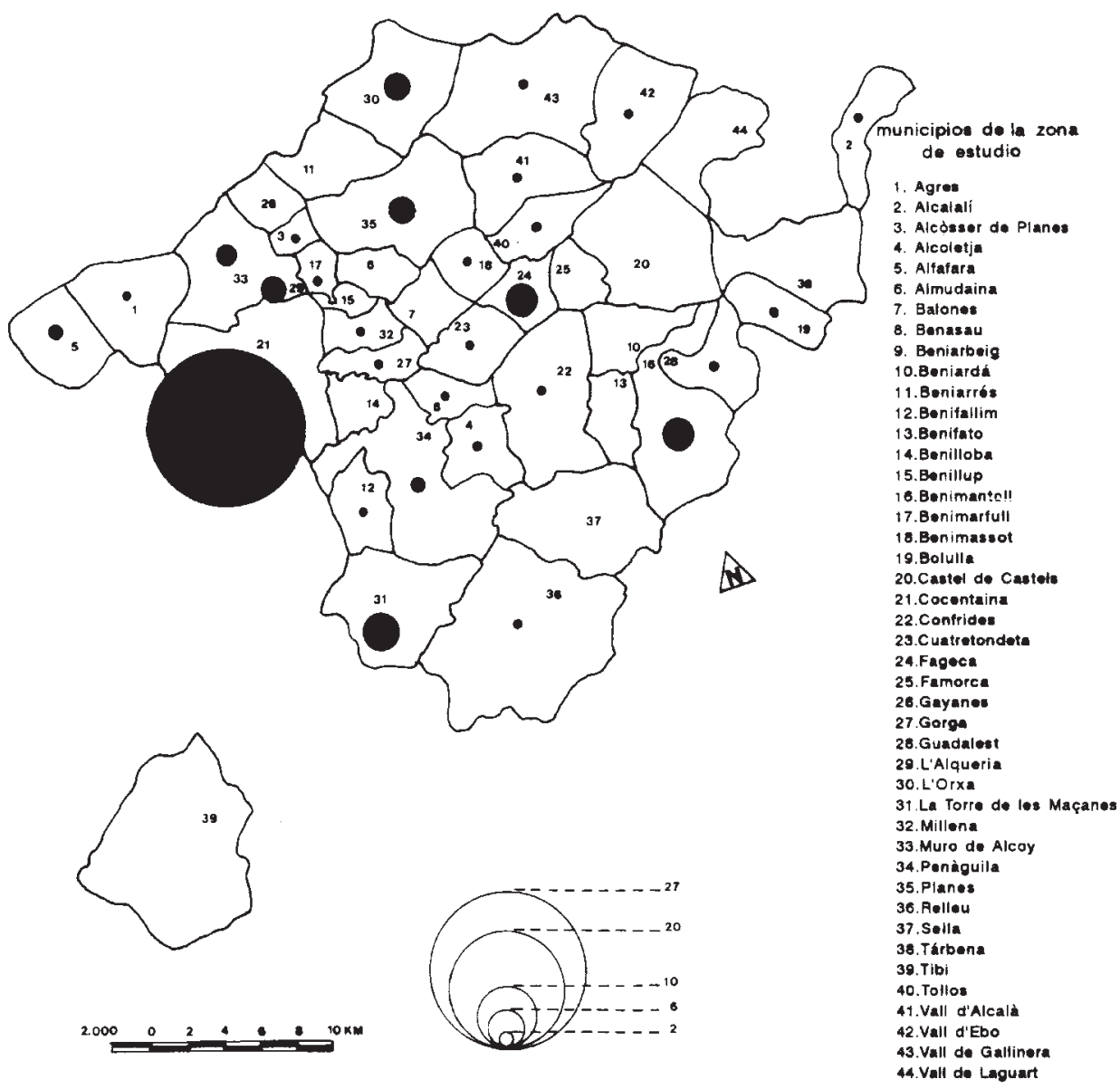

Figura 2. Número de proyectos subvencionados por municipio.

Tabla 2

PARTICIPACIÓN MUNICIPAL EN LA RELACIÓN DE INVERSIONES Y SUBVENCIONES EN ALOJAMIENTO Y RESTAURACIÓN

\begin{tabular}{|lrrrrrr|}
\hline PROYECTOS & INVERSIÓN & \multicolumn{1}{c}{$\%$} & SUBVENCIÓN & $\%$ & SOLICITUDES & $\%$ \\
\hline RESTAURANTE & 93.258 .470 & 25,99 & 33.905 .991 & 45,21 & 4 & 22,22 \\
PENSIONES & 4.464 .620 & 1,83 & 2.232 .310 & 4,00 & 1 & 12,50 \\
ALBERGUES & 101.960 .890 & 50,87 & 36.980 .445 & 53,66 & 2 & 28,57 \\
REH.VIVIENDA & 18.400 .000 & 59,37 & 7.360 .000 & 70,02 & 1 & 50,00 \\
ACAMPADAS & 11.995 .248 & 100,00 & 4.798 .099 & 100,00 & 2 & 100,00 \\
\hline
\end{tabular}

Fuente: CEDER. Datos a 30-IX-1994. Elaboración propia. 


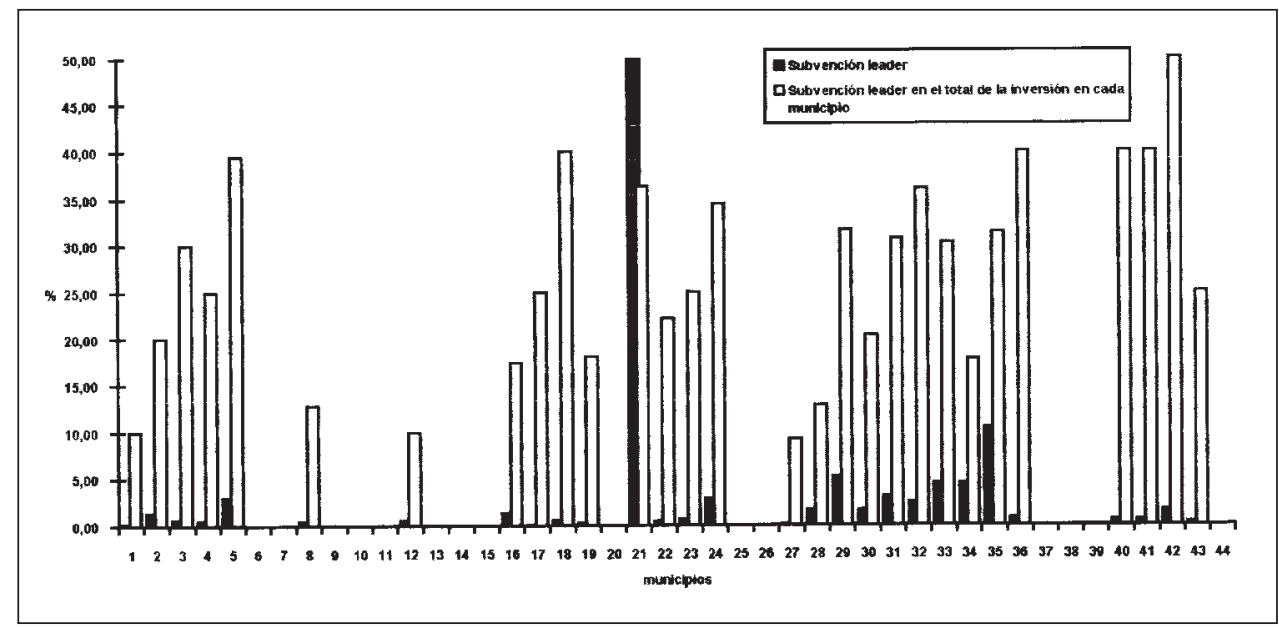

Fuente: CEDER. Datos a 30-IX-1994. Elaboración propia.

Por lo que se refiere a la distribución espacial de la aplicación de la iniciativa comunitaria LEADER, se ha de incidir en la elevada concentración de actuaciones en Cocentaina, que absorbe cerca de la mitad de la inversión total generada en La Montaña y de la subvención correspondiente a la misma. Este municipio ha centralizado la gestión del programa y aglutina los índices más elevados de población y actividad económica, así como las mayores disponibilidades de capital (ver fig. 2 y gráf. 3).

Esta situación resulta particularmente notoria, si tenemos en cuenta que el $65,91 \%$ de los municipios integrados en el CEDER presentan porcentajes de inversión inferiores al 1\%, valor que supera el 93\% si ampliamos el umbral al $6 \%$ de la inversión; únicamente el municipio de Planes supera el 10\% en inversión y subvención. Estos datos nos permiten confirmar la escasa homogeneidad de la distribución de las actuaciones en el territorio objeto de aplicación del LEADER, situación cuestionada por los municipios menos favorecidos, y que se agrava si tomamos en consideración que tres de los municipios relativamente más beneficiados integran la junta directiva encargada inicialmente de la gestión del LEADER. Esta coyuntura provocó ciertas disensiones que cristalizaron en la presentación de tres proyectos diferentes a la convocatoria del LEADER II, encabezados por Cocentaina, Planes y Callosa d'En Sarrià, si bien finalmente se aunaron de nuevo.

\section{Conclusiones}

A tenor de lo expuesto, en la valoración del LEADER de La Montaña sobresalen algunos puntos que han influido directamente en el resultado global del progra- 
ma, por supuesto, sin olvidar que se trata de una experiencia piloto que ha comenzado su funcionamiento en un espacio en el que la falta de infraestructuras y el escaso dinamismo económico ya comentado, han condicionado la agilidad en la gestión del grupo. Sin duda, los procesos más difíciles son los de puesta en marcha y creación de nuevas bases que permitan, a partir de ahí, construir un entramado sólido que facilite la irradiación de actividades económicas alternativas y complementarias a las tradicionales $\mathrm{y}$, por lo tanto, la diversificación productiva. Si tenemos en cuenta que La Montaña no disponía de condiciones mínimas que garantizaran la viabilidad del desarrollo turístico de la zona, esta labor resultaba ardua; ya que además de poseer unas condiciones naturales apropiadas, se requieren unos equipamientos básicos que aseguren la estancia de los turistas en la zona (alojamiento, restauración, etc.) y mantengan su interés por la misma (oferta de productos autóctonos, paisaje cuidado, ambiente tranquilo, contacto con la naturaleza, oferta complementaria).

A pesar de estas dificultades, la gestión del CEDER ha adolecido de ciertas deficiencias, que han hecho patente su inexperiencia y la escasa capacidad de cooperación con otras administraciones públicas, entidades financieras y centros de formación. Uno de los aspectos con una incidencia más negativa sobre el éxito del programa ha sido la insuficiente transmisión de la información, tanto desde el CEDER hacia algunos ayuntamientos, como desde éstos al resto de la población. El hecho de que el órgano de gestión del LEADER estuviera integrado exclusivamente por siete municipios de los 44 que forman la ZAM de La Montaña ha limitado notoriamente la coordinación entre todos ellos, ya que no se ha celebrado ninguna reunión conjunta en la que se expusieran el programa y sus objetivos. Algunos ayuntamientos tan sólo han recibido circulares informativas, pero no han asumido como propia la posibilidad de optar a los fondos leader; a la escasa comunicación se aúna el desinterés, en algunos casos, o las limitaciones inherentes al pequeño tamaño de los municipios, que generalmente disponen de unos medios financieros y humanos reducidos. Hubiera resultado, por ello, imprescindible la celebración de encuentros periódicos con representantes de los distintos ayuntamientos e, incluso, con agentes locales de estas zonas, para obtener una respuesta más adecuada de los mismos, despertar el interés hacia estas actuaciones e implicar realmente a la población en el proyecto de desarrollo local.

Otro aspecto conflictivo en relación a la gestión del CEDER, radica en la excesiva concentración de las subvenciones en el sector occidental de la Montaña, precisamente la zona aledaña a este centro, mientras que el extremo oriental las actuaciones han sido más puntuales. Asimismo es significativa la elevada cantidad de proyectos subvencionados a ayuntamientos que en los últimos meses se han visto favorecidos por las renuncias producidas, casi todas de particulares que han desistido de llevar a cabo la inversión ante el bajo porcentaje de la subvención 
otorgada; lo que viene a confirmar la escasez de agentes sociales con capacidad de actuación.

Por otro lado, la reducida ayuda a los particulares ha obligado a elevar el total de la inversión privada, lo que ha resultado problemático para los pequeños empresarios que la habían solicitado, al inducirles a desembolsos superiores a los previstos. Esta decisión de primar actuaciones de los ayuntamientos ocasiona cierta distorsión de los objetivos leader, que pretenden fundamentalmente potenciar una iniciativa empresarial permanente en la zona, ya de por sí bastante difícil de encontrar, y que además, concentra gran parte del presupuesto en acciones no productivas, que quizá hubieran tenido cabida en otras líneas de ayuda de organismos autonómicos o estatales ${ }^{15}$.

A estos hechos se suma la exigencia del CEDER de presentar facturas de las obras, y no sólo de los bienes muebles incorporados, en lugar de una certificación de obra. Este sistema supone unos costes superiores para el solicitante, dado que el IVA de las facturas absorbe buena parte de la subvención proporcionada por el LEADER, por lo que la ayuda pierde efectividad. La situación se complica, además, porque el beneficiario ha de realizar toda la inversión por adelantado, recurriendo con frecuencia a préstamos, mientras que los pagos correspondientes a las subvenciones sufren, en algunos casos, demoras. Los trámites necesarios para la presentación de solicitudes resultan, por otra parte, excesivamente complejos para el pequeño promotor, que ha echado en falta un mayor asesoramiento desde la oficina del CEDER, centro que no cuenta con los suficientes medios humanos y que no ha mantenido contactos frecuentes con el resto de entidades encargadas de aplicar los programas Leader aprobados en la Comunidad Valenciana, de los que podría haber extraído interesantes experiencias.

Podemos concluir que la aplicación del LEADER en la zona de la Montaña ha revestido un carácter peculiar respecto a los fines de la iniciativa de implantar una estrategia multisectorial de desarrollo y considerar la participación del turismo rural en la misma. Así, y en parte por las especiales condiciones de la zona de estudio, se ha destinado casi todo el presupuesto a esta actividad y, en menor medida, a la creación de pequeñas empresas artesanales, servicios locales y comercialización de la producción agraria local; y, sin embargo, no se ha enfocado al turismo vinculado a la agricultura, sino al turismo en una zona rural, ya que la mayor parte de los promotores no viven principalmente de la agricultura e, incluso, algunos son foráneos a la zona. A esto se añade el hecho de que, en algunos casos, se incorporan pautas propias del turismo costero.

15 Es interesante destacar el papel del PRU (Programa de Rehabilitación Urbana) de la Conselleria de Obras Públicas y Urbanismo, orientado al reacondicionamiento del patrimonio urbano. Este plan concede elevados porcentajes de ayuda a particulares para la rehabilitación de fachadas e interiores, y está siendo aprovechado por muchos municipios de la zona para renovar parte de su patrimonio inmobiliario degradado. 
Todos estos inconvenientes se pretenden solucionar en la aplicación del LEADER II, en el que la Conselleria de Agricultura actuará como organismo de control del CEDER, aprobando definitivamente los proyectos previamente seleccionados por este centro. Se contempla, por otra parte, la participación en la Junta Directiva de todos los municipios integrados en la zona de montaña, así como representantes de la Diputación y de varias entidades financieras, lo que permitirá un mayor consenso en la toma de decisiones. A este respecto, cabe señalar la posibilidad del desdoblamiento del CEDER en dos sedes planteada para la gestión del LEADER II, una de las cuales podría ubicarse en el sector oriental de La Montaña.

La formación, aspecto de gran importancia para el futuro desarrollo de iniciativas empresariales y la capacitación de personal, que ha sido relativamente descuidado en el LEADER I, presenta un sustancial cambio de orientación en el LEADER II, puesto que se prevee dirigir las actuaciones del CEDER a la colaboración con otros organismos que han venido desempeñando estas funciones, como es el caso de los CEIs (Centros de Empresas e Innovación), dependientes del IMPIVA, que han actuado en la Comunidad Valenciana como vivero de empresas desde los años 80 .

Este segundo programa, con un período de ejecución más dilatado, responde a una política de continuidad de las instancias europeas, en el sentido de consolidar las acciones ya emprendidas ${ }^{16}$; concretamente, pone el acento en la innovación aplicada al desarrollo rural y pretende potenciar aquellas líneas que complementen, de alguna manera, las indicaciones contenidas en el Marco Comunitario de Apoyo. Asimismo, incluye en estos programas de innovación un apartado dedicado a la conservación y mejora del medio ambiente y el entorno, de acuerdo con la filosofía de la PAC en este aspecto.

\section{Propuestas}

1. La implantación del turismo rural en La Montaña de Alicante debe tener en cuenta las características propias de la zona, en particular un potencial de recursos limitado, en el que el turismo rural puede actuar como alternativa no excluyente a las actividades económicas tradicionales, orientado básicamente hacia el ocio de proximidad de las áreas urbanas cercanas y al complemento del producto turístico litoral, dada la accesibilidad y proximidad desde la costa.

2. No hay que olvidar que cuando la iniciativa comunitaria plantea el desarrollo local, se está refiriendo a un desarrollo integrado, lo que implica la necesidad de considerar las relaciones del turismo rural con el resto de actividades económicas y, por supuesto, con el entramado socioeconómico y el medio natural.

16 De hecho, en la provincia de Alicante también se presentaron al segundo LEADER, otras tres candidaturas: La Vila Joiosa, la Vega Baja y el Alto Vinalopó, que, sin embargo, fueron desestimadas. 
3. En esta línea, dada la fragilidad del medio rural no se puede llevar a cabo un desarrollo turístico espontáneo, de ahí que las actuaciones a establecer en la zona exijan una labor de planificación territorial y sectorial previa para que el turismo pueda así contribuir a la dinamización económica de la zona; una planificación que evalúe sus recursos y valores paisajísticos y que debería recurrir a las directrices diseñadas por planes de rango superior, como pueda ser, para el ámbito de la Comunidad Valenciana, el futuro Plan Director de Espacios Turísticos elaborado por el Instituto Turístico Valenciano. Sin duda estos planes han de ser vinculantes para tener una efectividad real y garantizar el compromiso de los agentes sociales.

4. Sería conveniente, basándose en las peculiaridades de la zona, dotar de identidad propia a la oferta de ocio, que suponga la creación de productos específicos e innovadores para diferenciarlos del resto de productos de interior, a la vista del esfuerzo que están realizando en la promoción del turismo rural las distintas comunidades autónomas. Atendiendo a estos presupuestos sería conveniente diseñar y promocionar una marca global que identifique a todos los productos de La Montaña.

5. Estos planteamientos exigen, por tanto, la realización de estudios de la potencialidad del entorno y de la demanda y, asimismo, organizar convenientemente la oferta, ya que actualmente se caracteriza por su escasez y desconexión y por la carencia evidente de estrategias de promoción y comercialización. A este respecto, se han de promover equipamientos e infraestructuras adecuadas al consumo de productos típicamente rurales y evitar el simple traslado de las pautas seguidas en el turismo litoral.

6. Por último es importante hacer partícipe a toda la comunidad en un proyecto de conjunto, y conceder la suficiente importancia a la formación de profesionales relacionados con esta actividad y a la captación y preparación de agentes de desarrollo local.

\section{Bibliografía}

ARNALTE, E.: «La política de estructuras en la agricultura valenciana», en MARTÍNEZ SERRANO, J.A. y otros: Estructura económica de la Comunidad Valenciana, Biblioteca de Economía. Espasa-Calpe, Madrid, 1992, pp. 419-439.

BARDÓN FERNÁNDEZ, E.: «El turismo rural en España. Algunas iniciativas públicas» en Revista de Estudios Turísticos, 1987, pp. 63-76.

BARDÓN FERNÁNDEZ, E.: «Consideraciones sobre el turismo rural en España y medidas de desarrollo» en RET n ${ }^{\circ} 108,1990$, pp. 61-82.

BELTRÁN FERNÁNDEZ, C.: «El desarrollo rural y la iniciativa comunitaria LEADER en España», en Papeles de Economía Española, nº 60/61, 1994, pp. 226-228.

BOTE GÓMEZ, V: Turismo en espacio rural. Rehabilitación del patrimonio sociocultural y de la economía local. Editorial Popular. Madrid, 1988. 
CALATRAVA, J.: «El turismo rural como recurso endógeno en el desarrollo local: consideraciones teóricas y comentarios sobre las Alpujarras Altas Occidentales», en Desarrollo rural: Ejemplos Europeos. MAPA, IRYDA, 1992, pp. 67-91.

CIDEC y otros: «Turismo rural y agroturismo en España», número especial en Noticias de la Economía Pública, Social y Cooperativa, no 9, 1993.

CONSELLERIA DE INDUSTRIA, COMERCIO Y TURISMO: Memorias de actividades 1992 y 1993.

FRANCO, J.A.: «Balance de cinco años de aplicación de la legislación de estructuras comunitarias en la Comunidad Valenciana (1986-1991)», en Agriculturas y políticas agrarias en el sur de Europa. MAPA. Madrid, 1993, pp. 489-516.

GENERALITAT VALENCIANA: Turismo rural. Guía de recursos para cooperativas polivalentes y empresas. Elche, 1991.

GILBERT, D.C.: «Perspectivas de desarrollo del turismo rural», en Revista d'Estudis Autonòmics, $\mathrm{n}^{\circ} 13,1992$, pp. 167-193.

HUBERT, B.: «Nueva política comunitaria de estructuras agrarias y de desarrollo rural y la reforma de la PAC», en Agriculturas y políticas agrarias en el sur de Europa. MAPA. Madrid, 1993, pp. 209-226.

IVARS BAIDAL, J.A.: «¿Constituye el Turismo la mejor apuesta para el desarrollo rural?», comunicación presentada a las IV Jornadas de Geografía del Turismo: los turimos de interior. A.G.E. Toledo, 1995.

MARTÍ MARCO, M.R. y otros: Estudio para la aplicación del plan LEADER. IVERCEDER La Montaña, 1993.

PÉREZ YRUELA, M. y GIMÉNEZ GUERRERO, M.M.: «Desarrollo local y desarrollo rural: el contexto del programa LEADER», en Papeles de Economía Española, n $60 / 61,1994$, pp. 219-233.

VERA REBOLLO, J.F.: «Territorio, turismo y medio ambiente», en ¿Crisis del turismo? Las perspectivas en el nuevo escenario internacional. Instituto de Desarrollo Regional, Universidad de Sevilla-Universidad de Málaga, 1993, pp. 139-148.

YEPES, V.: «Turismo sostenible en el interior de la Comunidad Valenciana» en Papers de Turisme (en prensa). 